\title{
ON THE HARMONIC SUMMABILITY OF DOUBLE FOURIER SERIES
}

\author{
P. L. SHARMA
}

1. Suppose that the function $f(u, v)$ is integrable in the sense of Lebesgue, over the square $(-\pi, \pi ;-\pi, \pi)$ and is periodic with period $2 \pi$ in each variable. Let

$$
\begin{aligned}
\phi(u, v)= & \phi_{x}(u, v)=\frac{1}{4}[f(x+u, y+v)+f(x+u, y-v) \\
& +f(x-u, y+v)+f(x-u, y-v)-4 s] .
\end{aligned}
$$

Definition. The double sequence $\left\{s_{m, n}\right\}$ is said to be summable by Harmonic means, or summable $(H, 1,1)$ if

$$
\lim _{m \rightarrow \infty, n \rightarrow \infty} \frac{1}{\log m \log n} \sum_{l=0}^{m} \sum_{k=0}^{n} \frac{s_{m-l, n-k}}{(l+1)(k+1)}
$$

exists.

This is a particular case $p_{n}=1 /(n+1)$ of Norlund summability of a double sequence as defined by Herriot [4], Hille and Tamarkin [5] have proved the following theorem on the Harmonic Summability of Fourier Series:

Theorem A. The Fourier Series of the function $f(x)$ is summable $(H, 1)$ at the point $x$ at which

$$
\phi_{1}(t)=\int_{0}^{t}|\phi(u)| d u=o\left(\frac{t}{\log \frac{1}{t}}\right)
$$

where $\phi(t)=f(x+t)+f(x-t)-2 f(x)$.

An easy proof of this theorem is given by Prasad and Siddiqui [6].

We shall prove the theorem:

Theorem B. If

$$
\begin{aligned}
\Phi(u, v)= & \int_{0}^{u} d s \int_{0}^{v}|\phi(s, t)| d t=o\left(\frac{u v}{\log \frac{1}{u} \log \frac{1}{v}}\right), \\
& \int_{0}^{\pi} d t\left|\int_{0}^{u} \phi(s, t) d s\right|=O\left(\frac{u}{\log \frac{1}{u}}\right),
\end{aligned}
$$

Received by the Editors October 3, 1957. 


$$
\int_{0}^{\pi} d s\left|\int_{0}^{v} \phi(s, t) d t\right|=O\left(\frac{v}{\log \frac{1}{v}}\right)
$$

then the double Fourier Series of function $f(u, v)$ is summable $(H, 1,1)$ to the sum $s$ at $u=x$ and $v=y$.

This theorem is a generalization of Theorem A for double Fourier series and also is analogous to the theorem of Chow [1] for summability $(C, 1,1)$ of the double Fourier series.

2. We require the following lemmas:

Lemia 1. If $0<t<\pi$, then

$$
\left|\sum_{k=0}^{n} \frac{\cos (k+1) t}{k+1}\right|<A\left(1+\log \frac{1}{t}\right) .
$$

This is known [3].

Lemma 2. For all values of $n$ and $x$

$$
\left|\sum_{k=0}^{n} \frac{\sin (k+1) t}{k+1}\right| \leqq \frac{1}{2} \pi+1
$$

This is known [7].

Lemma 3. For $t$ such that $0 \leqq t \leqq 1 / n$

$$
\left|k_{n}(t)\right|=\left|\frac{1}{2 \pi \log n} \sum_{\gamma=0}^{n} \frac{1}{\gamma+1} \frac{\sin (n-\gamma+1 / 2) t}{\sin t / 2}\right|=O(n)
$$

where $k_{n}(t)$ is Harmonic Summability Kernel for Fourier series.

Proof.

$$
\begin{aligned}
\left|k_{n}(t)\right| & =\left|\frac{1}{2 \pi \log n} \sum_{\gamma=0}^{n} \frac{1}{\gamma+1} \frac{\sin (n-\gamma+1 / 2) t}{\sin t / 2}\right| \\
& =O\left(\frac{1}{\log n} \sum_{\gamma=0}^{n} \frac{1}{\gamma+1} \frac{(2 n-2 \gamma+1)|\sin t / 2|}{|\sin t / 2|}\right) \\
& =O\left(\frac{2 n+1}{\log n} \sum_{\gamma=0}^{n} \frac{1}{\gamma+1}\right) \\
& =O(n) .
\end{aligned}
$$

Lemma 4 . For $t$ such that $1 / n \leqq t \leqq \delta$. 


$$
\left|k_{n}(t)\right|=O\left[\frac{1}{t \log n}\{1+\log 1 / t\}\right]
$$

Proof.

$$
\begin{aligned}
k_{n}(t)= & \frac{1}{2 \pi \log n} \sum_{\gamma=0}^{n} \frac{1}{\gamma+1} \frac{\sin (n-\gamma+1 / 2) t}{\sin t / 2} \\
= & \frac{1}{2 \pi \log n \sin t / 2} \sum_{\gamma=0}^{n} \frac{1}{\gamma+1}\left\{\sin \left(n+\frac{3}{2}\right) t \cos (\gamma+1) t\right. \\
& \left.-\cos \left(n+\frac{3}{2}\right) t \sin (\gamma+1) t\right\} \\
= & \frac{1}{2 \pi \log n \sin t / 2}\left\{\sin \left(n+\frac{3}{2}\right) t \sum_{\gamma=0}^{n} \frac{\cos (\gamma+1) t}{\gamma+1}\right. \\
\left|k_{n}(t)\right|= & O\left[\frac{1}{t \log n}\left\{\left|\sum_{\gamma=0}^{n} \frac{\cos (\gamma+1) t}{\gamma+1}\right|+\left|\sum_{\gamma=0}^{n} \frac{\sin (\gamma+1) t}{\gamma+1}\right|\right\}\right] \\
= & O\left[\frac{1}{t \log n}\left\{A\left(1+\log \frac{1}{t}\right)+\frac{1}{2} \pi+1\right\}\right], \\
= & O\left[\frac{1}{t \log n}\left\{1+\log \frac{1}{t}\right\}\right] .
\end{aligned}
$$

Lemma 5. For $t$ such that $\delta \leqq t \leqq \pi$

$$
\left|k_{n}(t)\right|=O\left[\frac{1}{t \log n}\right] \text {. }
$$

Proof. Applying Abel's transformation, we have

$$
\begin{aligned}
\left|k_{n}(t)\right|= & O\left[\frac{1}{t \log n} \sum_{\gamma=0}^{n-1}\left(\frac{1}{\gamma-1}-\frac{1}{\gamma-2}\right)\right. \\
& \left.\cdot \frac{\sin [(n+1 / 2)+v-1 / 2] t \sin v t / 2}{\sin t / 2}\right]+O\left[\frac{1}{n t \log n}\right] \\
= & O\left[\frac{1}{t \log n}\right] .
\end{aligned}
$$

3. Proof of the theorem 


$$
\begin{aligned}
& \pi^{2} \sigma_{m, n}=\int_{0}^{\pi} \int_{0}^{\pi} \phi(u, v) k_{m}(u) k_{n}(v) d u d v \\
& =\left(\int_{0}^{\delta} \int_{0}^{\tau}+\int_{0}^{\delta} \int_{\tau}^{\pi}+\int_{\delta}^{\pi} \int_{0}^{\tau}+\int_{\delta}^{\pi} \int_{\tau}^{\pi}\right) \phi(u, v) k_{m}(u) k_{n}(v) d u d v \\
& \text { where } 1 / m<\delta<\pi, 1 / n<\tau<\pi \\
& =I_{1}+I_{2}+I_{3}+I_{4} \text { say. } \\
& \left|I_{4}\right|=O\left(\frac{1}{\log m \log n} \int_{\delta}^{\pi} \int_{\tau}^{\pi} \frac{|\phi(u, v)|}{u v} d u d v\right) \\
& =o(1) \text { by the help of Lemma } 1 \text {. } \\
& I_{3}=\int_{\delta}^{\pi} k_{m}(u) d u \int_{0}^{\tau} \phi(u, v) k_{n}(u) d v \\
& =\int_{\delta}^{\pi} k_{m}(u) d u \int_{0}^{1 / n} \phi(u, v) k_{n}(v) d v+\int_{\delta}^{\pi} k_{m}(u) d u \int_{1 / n}^{\tau} \phi(u, v) k_{n}(u) d v \\
& =I_{3,1}+I_{3,2} \text { say. }
\end{aligned}
$$

By Lemmas 3, 4 and the theorem,

$$
\begin{aligned}
& \left|I_{3,1}\right|=O\left[\frac{n}{\log m} \int_{0}^{1 / n}|\phi(u, v)| d v\right] \\
& =o(1) \text {. } \\
& \left|I_{3,2}\right|=O\left[\frac{1}{\log m} \int_{1 / n}^{\tau}|\phi(u, v)| \frac{1}{v \log n}\left(1+\log \frac{1}{v}\right) d v\right] \\
& =O\left[\frac{1}{\log m \log n} \int_{1 / n}^{\tau}|\phi(u, v)| \frac{d v}{v}\right] \\
& +O\left[\frac{1}{\log m \log n} \int_{1 / n}^{\tau}|\phi(u, v)| \frac{1}{v} \log \frac{1}{v} d v\right] \\
& =\left|I_{3,2,1}\right|+\left|I_{3,2,2}\right| \text { say. } \\
& \left|I_{3,2,1}\right|=O\left[\frac{1}{\log m \log n}\left[\Phi(u, v) \frac{1}{v}\right]_{1 / n}^{\tau}+\frac{1}{\log m \log n} \int_{1 / n}^{\tau} \Phi(u, v) \frac{d v}{v^{2}}\right] \\
& =O\left[\frac{1}{\log m \log n}\right]+o\left[\frac{1}{\log m \log n} \int_{1 / n}^{\tau} \frac{d v}{v \log 1 / v}\right] \\
& =o(1)+o\left[\frac{\log \log 1 / v}{\log n}\right]_{1 / n}^{\tau} \\
& =o(1) \text {. }
\end{aligned}
$$


1958]

HARMONIC SUMMABILITY OF DOUBLE FOURIER SERIES

983

$$
\begin{aligned}
\left|I_{3,2,2}\right|= & O\left[\frac{1}{\log m \log n} \int_{1 / n}^{\tau}|\phi(u, v)| \frac{1}{v} \log \frac{1}{v} d v\right] \\
= & O\left[\frac{1}{\log m \log n}\left[\Phi(u, v) \frac{1}{v} \log \frac{1}{v}\right]_{1 / n}^{\tau}\right. \\
& \left.+\frac{1}{\log m \log n} \int_{1 / n}^{\tau} \Phi(u, v)\left\{1+\log \frac{1}{v}\right\} \frac{d v}{v^{2}}\right] \\
= & o(1)+O\left(\frac{1}{\log m \log n} \int_{1 / n}^{\tau} \frac{\Phi(u, v)}{v^{2}} d v\right) \\
& +O\left(\frac{1}{\log m \log n} \int_{1 / n}^{\tau} \frac{\Phi(u, v)}{v^{2}} \log \frac{1}{v} d v\right) \\
= & o\left(\frac{1}{\log n} \int_{1 / n}^{\tau} \frac{d v}{v \log 1 / v}\right)+o\left(\frac{1}{\log n} \int_{1 / n}^{\tau} \frac{d v}{v}\right) \\
= & o\left(\left[\frac{\log \log 1 / v}{\log n}\right]_{1 / n}^{\tau}\right)+o\left(\left[\frac{\log 1 / v}{\log n}\right]_{1 / n}^{\tau}\right) \\
= & o(1) .
\end{aligned}
$$

Thus $\left|I_{3}\right|=o(1)$. Similarly $\left|I_{2}\right|=o(1)$.

Let us evaluate $I_{1}$.

$$
\begin{aligned}
I_{1}=\left(\int_{0}^{1 / m} \int_{0}^{1 / n}+\int_{0}^{1 / m} \int_{1 / n}^{\tau}+\int_{1 / m}^{\delta} \int_{0}^{1 / n}+\int_{1 / m}^{\delta} \int_{1 / n}^{\tau}\right) & \cdot \phi(u, v) k_{m}(u) k_{n}(v) d u d v \\
=I_{1,1}+I_{1,2}+I_{1,3}+I_{1,4}, \text { say. } & \\
\left|I_{1,1}\right| & =O\left(\int_{0}^{1 / m} \int_{0}^{1 / n}|\phi(u, v)|\left|k_{m}(u)\right|\left|k_{n}(v)\right| d u d v\right) \\
& =O\left(\int_{0}^{1 / m} \int_{0}^{1 / n}|\phi(u, v)| m n d u d v\right) \\
& =o(1) . \\
\left|I_{1,2}\right| & =O\left[\int_{0}^{1 / m} m d u \int_{1 / n}^{\tau} \phi(u, v) k_{n}(v) d v\right] \\
& =O\left[\int_{1 / n}^{\tau} \phi(u, v) k_{n}(v) d v\right] \\
& =o(1) \text { as } I_{3,2} .
\end{aligned}
$$


Thus $\left|I_{1,2}\right|=o(1)$. Similarly $\left|I_{1,3}\right|=o(1)$.

$$
\begin{aligned}
& I_{1,4}=\int_{1 / m}^{\delta} \int_{1 / n}^{\tau} \phi(u, v) k_{m}(u) k_{n}(v) d u d v . \\
& \left|I_{1,4}\right|=O\left[\int_{1 / m}^{\delta} \int_{1 / n}^{\tau}|\phi(u, v)| \frac{1}{u \log m}\left\{1+\log \frac{1}{u}\right\} \frac{1}{v \log n}\right. \\
& \left.\cdot\left\{1+\log \frac{1}{v}\right\} d u d v\right] \\
& =O\left[\int_{1 / m}^{\delta} \int_{1 / n}^{\tau}|\phi(u, v)| \frac{1}{u \log m} \cdot \frac{1}{v \log n} d u d v\right] \\
& +O\left[\int_{1 / m}^{\delta} \int_{1 / n}^{\tau}|\phi(u, v)| \frac{1}{u \log m} \log \frac{1}{u} \frac{1}{v \log n} d u d v\right] \\
& +O\left[\int_{1 / m}^{\delta} \int_{1 / n}^{\tau}|\phi(u, v)| \frac{1}{u \log m} \frac{1}{v \log n} \log \frac{1}{v} d u d v\right] \\
& +O\left[\int_{1 / m}^{\delta} \int_{1 / n}^{\tau}|\phi(u, v)| \frac{1}{u \log m} \log \frac{1}{u} \frac{1}{v \log n} \log \frac{1}{v} d u d v\right] \\
& =\left|I_{1,4,1}\right|+\left|I_{1,4,2}\right|+\left|I_{1,4,3}\right|+\left|I_{1,4,4}\right| \text {. } \\
& \left|I_{1,4,1}\right|=O\left[\int_{1 / m}^{\delta} \frac{1}{u \log m} d u \int_{1 / n}^{\tau} \frac{|\phi(u, v)|}{v \log n} d v\right] \\
& =O\left[\frac{1}{\log n} \int_{1 / n}^{r} \frac{|\phi(u, v)|}{v} d v\right] \\
& =o(1) \text { by the help of } I_{3,2,1} \text {. } \\
& \left|I_{1,4,2}\right|=O\left[\int_{1 / n}^{\tau} \frac{1}{v \log n} d v \int_{1 / m}^{\delta}|\phi(u, v)| \frac{1}{u \log m} \log \frac{1}{u} d u\right] \\
& =O\left[\frac{1}{\log m} \int_{1 / m}^{\delta}|\phi(u, v)| \frac{1}{u} \log \frac{1}{u} d u\right] \\
& =o(1) \text { by } I_{3,2,2} \text {. } \\
& \left|I_{1,4,3}\right|=o(1) \text { as }\left|I_{1,4,2}\right| \text {. } \\
& \left|I_{1,4,4}\right|=O\left[\int_{1 / m}^{\delta} \int_{1 / n}^{\tau}|\phi(u, v)| \frac{1}{u \log m} \cdot \log \frac{1}{u} \frac{1}{v \log n} \log \frac{1}{v} d u d v\right] .
\end{aligned}
$$

By partial integration for double integral we have [2], 


$$
\begin{aligned}
& \Phi(\delta, \tau) \frac{1}{\delta \log m} \log \frac{1}{\delta} \frac{1}{\delta \log n} \log \frac{1}{\delta} \\
& -\frac{1}{\tau \log n} \log \frac{1}{\tau} \int_{1 / m}^{\delta} \Phi(u, \tau) \frac{1-\log 1 / u}{u^{2} \log m} d u \\
& -\frac{1}{\delta \log m} \log \frac{1}{\delta} \int_{1 / n}^{\tau} \Phi(\delta, v) \frac{1-\log 1 / v}{v^{2} \log n} d v \\
& +\int_{1 / m}^{\delta} \int_{1 / n}^{r} \Phi(u, v) \frac{(1-\log 1 / u)(1-\log 1 / v)}{u^{2} v^{2} \log m \log n} d u d v \\
& =L_{1}+L_{2}+L_{3}+L_{4} . \\
& L_{1}=o(1) \text {. } \\
& L_{2}=o\left(\int_{1 / m}^{\delta} \Phi(u, \tau) \frac{1}{u^{2} \log m} d u\right)+o\left(\int_{1 / n}^{\tau} \Phi(u, \tau) \frac{\log 1 / u}{u^{2}} d u\right) \\
& =o(1) \text { by } I_{3,2,1} \text { and } I_{3,2,2} \text {. } \\
& L_{3}=o(1) \text { as in } L_{2} \text {. } \\
& L_{4}=\int_{1 / m}^{\delta} \int_{1 / n}^{\tau} \frac{\Phi(u, v) d u d v}{u^{2} v^{2} \log m \log n} \\
& -\int_{1 / m}^{\delta} \int_{1 / n}^{\tau} \frac{\Phi(u, v) \log (1 / u) d u d v}{u^{2} v^{2} \log m \log n} \\
& -\int_{1 / m}^{\delta} \int_{1 / n}^{\tau} \frac{\Phi(u, v) \log (1 / v) d u d v}{u^{2} v^{2} \log m \log n} \\
& +\int_{1 / m}^{\delta} \int_{1 / n}^{\tau} \frac{\Phi(u, v) \log (1 / u) \log (1 / v) d u d v}{u^{2} v^{2} \log m \log n} \\
& =L_{4,1}+L_{4,2}+L_{4,3}+L_{4,4} \text {. } \\
& L_{4,1}=o\left(\int_{1 / m}^{\delta} \frac{d u}{u \log 1 / u} \int_{1 / n}^{\tau} \frac{d v}{v \log 1 / v}\right) \\
& =o(1) \text {. } \\
& L_{4,2}=\left(\int_{1 / m}^{\delta} \int_{1 / n}^{\tau} \frac{1}{u} \frac{1}{v \log 1 / v} \cdot \frac{1}{\log m \log n} d u d v\right) \\
& =o\left(\int_{1 / m}^{\delta} \frac{d u}{u \log m} \int_{1 / n}^{\tau} \frac{d u}{v \log 1 / v}\right) \\
& =o(1) \text {. }
\end{aligned}
$$


Similarly $L_{4,3}=o(1)$.

$$
\begin{aligned}
L_{4,4} & =o\left(\int_{1 / m}^{\delta} \frac{d u}{u \log m} \int_{1 / n}^{\tau} \frac{d v}{v \log n}\right. \\
& =o(1) .
\end{aligned}
$$

Thus the proof is complete.

I am much indebted to Dr. M. L. Misra for his kind help and guidance in the preparation of this paper.

\section{REFERENCES}

1. Y. S. Chow, On the cesaro summability of double Fourier series, Tôhoku Math. J. vol. 5 (1953) pp. 277-283.

2. J. J. Gergen, Convergence criteria for double Fourier series, Trans. Amer. Math. Soc. vol. 35 (1933) pp. 29-63.

3. Hardy and Rogosinki, Proc. Cambridge Philos. Soc. vol. 43 (1947) pp. 10-25.

4. J. G. Herriot, The Nörlund summability of double Fourier series, Trans. Amer. Math. Soc. vol. 52 (1942) pp. 72-94.

5. Hille and Tamarkin, On the summability of Fourier series, Trans. Amer. Math. Soc. vol. 34 (1932) pp. 757-783.

6. B. N. Prasad and Siddiqui, Harmonic summability of Fourier series, Proc. Indian Acad. Sci. vol. 28 (1948) pp. 527-531.

7. E. C. Titchmarsh, Theory of functions, p. 40.

University of SAUgar, SAgar, India 